\title{
PAPER
}

\section{A randomised placebo controlled exploratory study of vitamin B-12, lofepramine, and L-phenylalanine (the "Cari Loder regime") in the treatment of multiple sclerosis}

\author{
D T Wade, C A Young, K R Chaudhuri, D L W Davidson
}

J Neurol Neurosurg Psychiatry 2002;73:246-249

\begin{abstract}
Objective: To determine whether combination therapy with lofepramine, L-phenylalanine, and intramuscular vitamin B-12 (the "Cari Loder regime") reduces disability in patients with multiple sclerosis.

Methods: A placebo controlled, double blind, randomised study carried out in five United Kingdom centres on outpatients with clinically definite multiple sclerosis, measurable disability on Guy's neurological disability scale (GNDS), no relapse in the preceding six months, and not on antidepressant drugs. Over 24 weeks all patients received vitamin B-12, $1 \mathrm{mg}$ intramuscularly weekly, and either lofepramine $70 \mathrm{mg}$ and L-phenylalanine $500 \mathrm{mg}$ twice daily, or matching placebo tablets. Outcome was assessed using the GNDS, the Kurtzke expanded disability status scale; the Beck depression inventory, the Chalder fatigue scale, and the Gulick MS specific symptom scale.

Results: 138 patients were entered, and two were lost from each group. There was no statistically significant difference between the groups at entry or at follow up. Analysis of covariance suggested that treated patients had better outcomes on four of the five scales used. Both groups showed a reduction of 2 GNDS points within the first two weeks, and when data from all time points were considered, the treated group had a significant improvement of 0.6 GNDS points from two weeks onwards.

Conclusions: Patients with multiple sclerosis improved by 2 GNDS points after starting vitamin B-12 injections. The addition of lofepramine and L-phenylalanine added a further 0.6 points benefit. More research is needed to confirm and explore the significance of this clinically small difference.
\end{abstract}

5 November 2001

Accepted 28 March 2002

...................

$\mathrm{P}$ atients with multiple sclerosis often approach their doctors with suggestions and requests for novel therapy because there are so few treatments of proven efficacy. There is some evidence to support the use of disease modifying drugs such as $\beta$ interferons and azathioprine, although the benefits are small. ${ }^{1}$ There is more evidence in support of symptomatic treatments such as amantadine for fatigue ${ }^{2}$ and baclofen for spasticity. Rehabilitation by a multidisciplinary specialist team may also be beneficial. ${ }^{34}$ Nonetheless patients will continue to seek alternative treatments.

In 1996 Ms Cari Loder published a book ${ }^{5}$ espousing one such treatment, the combination of oral lofepramine and L-phenylalanine with vitamin B-12 injections. One element of this, lofepramine, had been proposed by a Swedish group, but only uncontrolled data were published. ${ }^{6}$

The scientific rationale is sparse. One paper has suggested that multiple sclerosis may follow from reduced central noradrenaline levels, and reported that "approximately $75 \%$ of [300] patients experience a substantial improvement" after a combination of tri- or tetracyclic antidepressants and L-DOPA. ${ }^{6}$ The current regime may increase noradrenaline levels-lofepramine limits noradrenaline uptake at synapses, and L-phenylalanine is a precursor of noradrenaline. We are unaware of any other supportive evidence or theory. No phase I or phase II studies were undertaken.

Our aim in this study was primarily to discover whether there was sufficient evidence of reduction in disability to support further investigation in detail. Secondary aims were to monitor possible side effects and to explore effects on a range of specific symptoms related to multiple sclerosis. In this exploratory study we investigated the dose regime proposed, ${ }^{5}$ to determine if benefits occurred that were worthy of further investigation. In the absence of any pilot trials, our study included any patient who had measurable disability, in

contrast to most current research which generally selects less disabled patients or other subgroups. This study is also one of the first major trials of a drug intervention to use a specific disability measure ${ }^{7-9}$ as its primary outcome.

\section{METHODS}

We used a double blind, randomised, placebo controlled design. All patients were given vitamin B-12 $1000 \mu \mathrm{g}$ intramuscularly weekly for 24 weeks. One group of patients was randomised to receive lofepramine $70 \mathrm{mg}$ twice daily and L-phenylalanine $500 \mathrm{mg}$ twice daily (the combination group), and the remainder were given matching placebo tablets (the control group).

Patients were included if they had clinically definite multiple sclerosis, ${ }^{10}$ were aged between 18 years and 65 years, gave written informed consent and were willing and able to comply with the follow up schedule, and had significant disability. Disability was defined as a score of 3.0 or more on the Kurtzke extended disability status scale (EDSS) ${ }^{11}$ and $2 / 5$ or more in two of eight domains from the Guy's neurological disability scale (GNDS). ${ }^{79}$ The eight domains were: fatigue, mobility, bladder, bowel, mental state, vision, speech, and swallowing.

Patients were excluded for the following reasons:

- if they had a relapse (clinically determined) or had taken steroids within the previous six months;

- if they were currently taking antidepressant drugs or were using hyperbaric oxygen;

- if they had used the trial treatment before;

- if they were hypertensive or had cardiac disease;

- if they were pregnant or not practising a reliable form of contraception; 


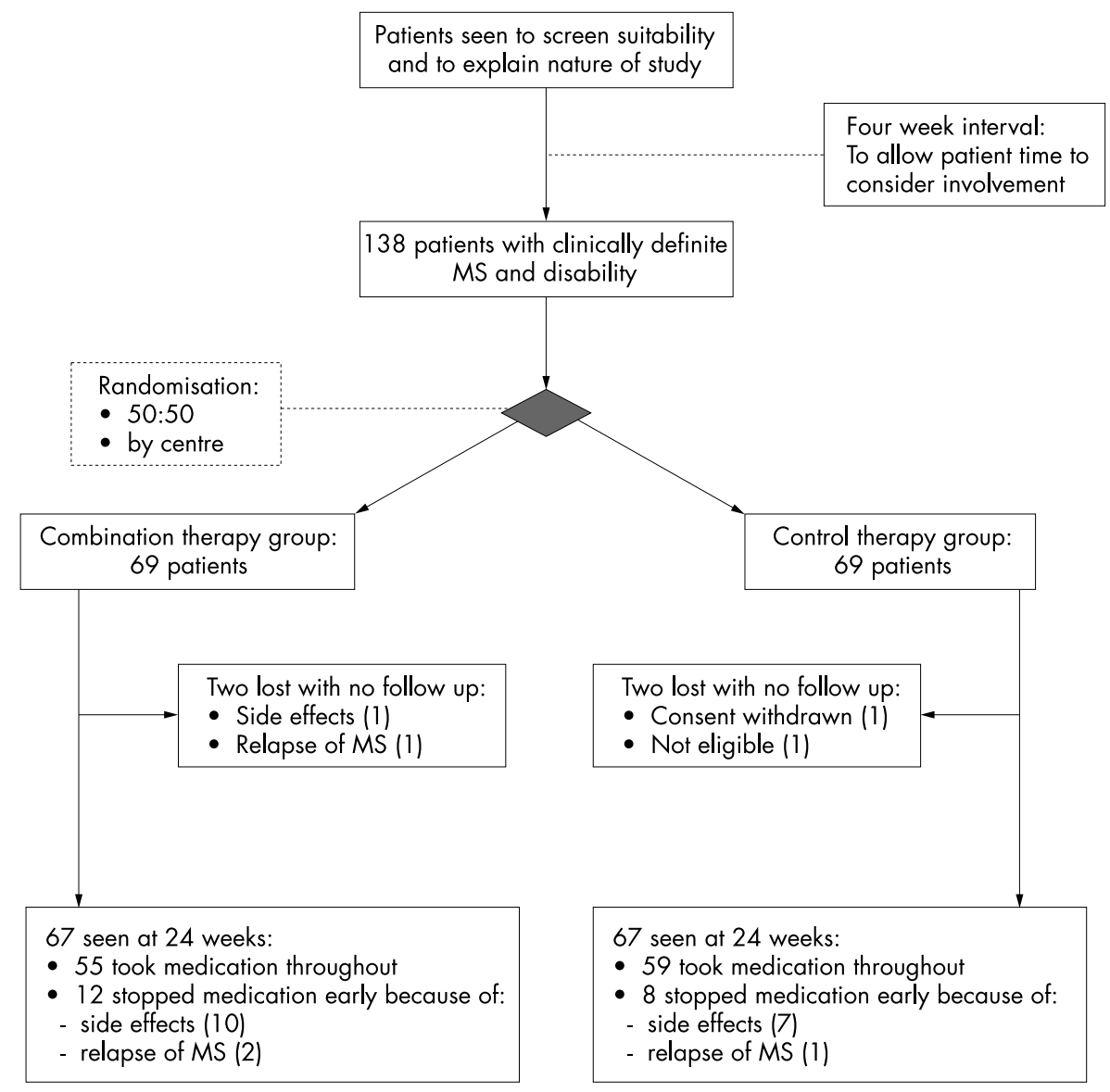

Figure 1 Flow diagram of the study.

- if they had any contraindication to taking any component of the treatment.

In the pretrial visit, eligibility was assessed and the trial design explained. In addition, there was a careful discussion of the low level of available evidence concerning benefits and risks.

The pretrial screening protocol included: a physical examination and assessment on Kurtzke EDSS ${ }^{11}$ and GNDS, ${ }^{7}$ an ECG, blood pressure measurement, and a blood sample for full blood count, urea and electrolytes, and vitamin B-12 level. Patients were given four weeks to consider their decision and if they agreed to continue then they had a baseline assessment and were given a diary to record changes noted over the first two weeks. They were then seen at weeks 2, 4, 12, and 24 .

Once a patient was registered, randomisation was undertaken centrally by Scotia Pharmaceuticals using a computer randomisation procedure. Patients were randomised within centres, but no other balancing factors were taken into account.

The formal assessments used were the Kurtzke EDSS, ${ }^{11}$ the GNDS, ${ }^{79}$ the Beck depression inventory, ${ }^{12}$ the Chalder fatigue scale, ${ }^{13}$ and the Gulick multiple sclerosis specific symptom scale. ${ }^{14}$ The last four were recorded at each assessment point, but the Kurtzke EDSS was only recorded at baseline and at 24 weeks.

The primary outcome measures were first, the change in the total score on the GNDS from entry to 24 weeks, and second, the cumulative benefit over time on the GNDS, taking into account scores at each assessment.

\section{Statistics}

Data were collected by Scotia Pharmaceuticals. Each centre obtained permission from their local ethics committee for the

\begin{tabular}{|c|c|c|}
\hline & $\begin{array}{l}\text { Combination group } \\
(\mathrm{n}=69)\end{array}$ & $\begin{array}{l}\text { Control group } \\
(\mathrm{n}=69)\end{array}$ \\
\hline Age (years) & $44.8(9.8)$ & $47.8(9.4)$ \\
\hline Age range (years) & 22 to 64 & 21 to 64 \\
\hline Male:female & $18: 51$ & $23: 46$ \\
\hline \multicolumn{3}{|l|}{ Type of MS: } \\
\hline Relapsing/remitting & 17 & 18 \\
\hline Primary progressive & 22 & 22 \\
\hline Secondary progressive & 30 & 29 \\
\hline Duration of MS (months) & $108.8(96.6)$ & $127.3(102.9)$ \\
\hline \multicolumn{3}{|l|}{ Kurtzke EDSS score } \\
\hline $0-2.5$ & 0 & 1 * \\
\hline $3.0-5.0$ & 22 & 13 \\
\hline $5.5-7.5$ & 34 & 44 \\
\hline $8.0-10$ & 13 & 11 \\
\hline \multicolumn{3}{|l|}{ GNDS score } \\
\hline Mean (SD) & $19.9(7.8)$ & $19.9(7.3)$ \\
\hline Range (median) & 5 to $39(19)$ & 6 to 40 (19) \\
\hline Beck score & $11.6(9.2)[n=66]$ & $11.7(8.7)$ \\
\hline MS symptoms score & $52.4(15.4)[\mathrm{n}=64]$ & $49.3(14.2)[n=65]$ \\
\hline MS ADL score & $47.1(16.0)[n=66]$ & $46.7(17.0)[n=66]$ \\
\hline Fatigue scale & $3.9(3.4)[n=68]$ & $3.4(3.5)[\mathrm{n}=67]$ \\
\hline \multicolumn{3}{|l|}{ Centre } \\
\hline A & 25 & 24 \\
\hline B & 15 & 16 \\
\hline C & 13 & 13 \\
\hline D & 13 & 12 \\
\hline $\mathrm{E}$ & 3 & 4 \\
\hline
\end{tabular}

Values are $\mathrm{n}$ or mean (SD).

*Protocol violator who was not followed up.

ADL, activities of daily living; EDSS, extended disability status scale; GNDS, Guy's neurological disability scale; MS, multiple sclerosis. 
Table 2 Change in scores at 24 weeks

\begin{tabular}{lll}
\hline & $\begin{array}{l}\text { Combination group } \\
(\mathrm{n}=67)\end{array}$ & $\begin{array}{l}\text { Control group } \\
(\mathrm{n}=67)\end{array}$ \\
\hline $\begin{array}{l}\text { GNDS score } \\
\begin{array}{l}\text { GNDS change: range } \\
\text { (median) }\end{array}\end{array}$ & $-3.6(5.4)$ & $-2.3(4.7)$ \\
$\begin{array}{l}\text { Beck depression } \\
\text { inventory }\end{array}$ & +8 to $-19(-3)$ & +12 to $-12(-2)$ \\
$\begin{array}{l}\text { Multiple sclerosis } \\
\text { symptom score }\end{array}$ & $-4.6(8.4)[\mathrm{n}=66]$ & $-3.3(7.2)$ \\
$\begin{array}{l}\text { Multiple sclerosis } \\
\text { disability score }\end{array}$ & $-8.1(14.2)[\mathrm{n}=64]$ & $-3.7(10.4)[\mathrm{n}=65]$ \\
$\begin{array}{l}\text { Fatigue scale } \\
\text { Kurtzke EDSS }\end{array}$ & $-2.1(3.3)[\mathrm{n}=66]$ & $0.8(8.3)[\mathrm{n}=66]$ \\
\hline
\end{tabular}

Values are mean (SD) unless stated.

EDSS, extended disability status scale; GNDS, Guy's neurological disability scale.

study. Initial power calculations using published data ${ }^{15}$ and unpublished data supplied by Dr Sharrack suggested that 60 patients would be needed in each group to detect a difference of three points on the GNDS. We aimed to recruit 140 patients, assuming a $10 \%$ dropout rate.

The primary statistical analyses compared the observed data between the two groups using appropriate parametric ( $t$ test) or non-parametric ( $\chi^{2}$ and Mann-Whitney) tests. All data were included, using the last observation carried forward if final data were missing (that is, an intention to treat analysis).

The effect of active treatment on the GNDS score was examined by fitting a linear repeated measurements model to the post-treatment data. The covariates (other than the treatment group) included in the final part of this model were: centre, multiple sclerosis disease type, smoking status (because anecdotal evidence suggested smokers responded well), sex, and baseline GNDS score. Preliminary data analysis suggested that a "split plot" (constant correlations) model was appropriate for these data, fitted by including a single random effect for subject. The model was fitted using the "lme" routine of S-Plus 2000. Two possible forms of treatment effect were tested. First, a model was fitted which investigated an increasing effect of treatment over time. However, this was rejected in favour of the second, simpler model which contained only which treatment group the patient was in.

Further analyses were undertaken to investigate two subsidiary questions. First, because there may have been unexpected differences between the groups, analysis of covariance was used to allow for any differences using the patient's initial score and centre as covariates. Second, given that some patients stopped the treatment before completion, data from patients who took the treatment throughout were compared $(\mathrm{n}=113)$.

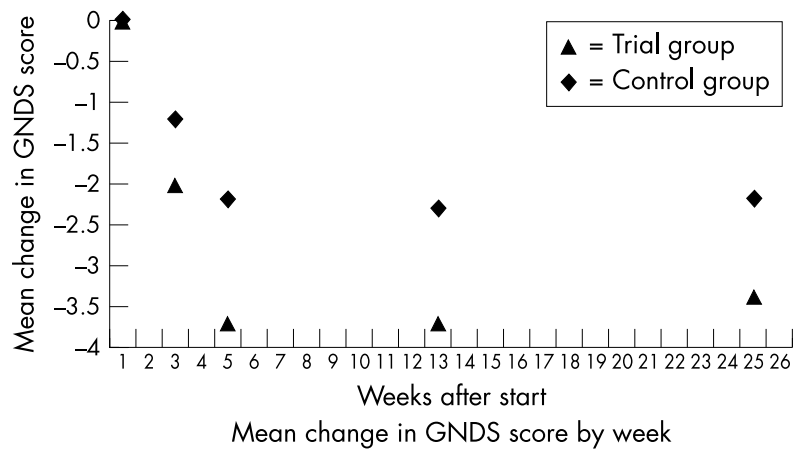

Figure 2 Mean change in GNDS score by week.

\section{RESULTS}

In all, 138 patients were entered into the study. Figure 1 shows the flow of patients through the study. The commonest reason for stopping the treatment was the occurrence of new symptoms, which were often those of the underlying disease. Only four patients withdrew from follow up. Table 1 shows the baseline characteristics of the two groups. There were no statistically significant differences between the groups at entry.

At 24 weeks there was a reduction in the mean GNDS score (representing an improvement) in both groups, with little change in the Kurtzke score (table 2; fig 2). The changes in scores were small in all measures, and none of the differences between the groups was statistically significant. The improvement seen over the first two weeks was not significant (paired $t$ test).

Analysis of the repeated measures of the GNDS using the linear model showed a benefit of 0.6 GNDS units in favour of the active treatment $(p=0.03)$, and the data suggest that this benefit occurred within two weeks and remained constant thereafter.

Analysis of covariance of the change scores in all patients (that is, an intention to treat analysis) showed a possible effect of combination therapy in reducing fatigue by a small amount, but no other benefit was found (table 3). Analysis of covariance restricted to treatment compliant patients also showed that combination therapy might reduce multiple sclerosis related symptoms (Gulick scale ${ }^{14}$ ) and reduce deterioration on the Kurtzke scale ${ }^{11}$ (table 3).

The majority of patients reported many adverse events, most of which were part of the normal fluctuation in multiple sclerosis symptoms. However, patients taking the combination treatment did experience more of the symptoms expected from the drugs (table 4), suggesting that the medication had some physiological effect.

Table 3 Change in scores (analysis of covariance)

\begin{tabular}{llll}
\hline Measure & Combination group & Control group & Mean difference \\
\hline GNDS, all follow up & $-4.09(-5.25$ to -2.94$)$ & $-2.93(-4.09$ to -1.77$)$ & $-1.16(-2.75$ to 0.43$)$ \\
GNDS, treatment compliant & $-4.56(-5.75$ to -3.37$)$ & $-3.19(-4.36$ to -2.02$)$ & $-1.37(-3.00$ to 0.27$)$ \\
Kurtzke, all follow up & $-0.01(-0.17$ to 0.15$)$ & $0.16(0.00$ to 0.32$)$ & $-0.17(-0.39$ to 0.05$)$ \\
Kurtzke, treatment compliant & $-0.10(-0.27$ to 0.06$)$ & $0.13(-0.03$ to 0.30$)$ & $-0.24(-0.46 \text { to }-0.01)^{\text {a }}$ \\
Beck, all follow up & $-4.87(-6.48$ to -3.27$)$ & $-3.44(-5.04$ to -1.85$)$ & $-1.43(-3.62$ to 0.76$)$ \\
Beck, treatment compliant & $-6.24(-7.57$ to -4.90$)$ & $-4.71(-6.01$ to -3.41$)$ & $-1.53(-3.35$ to 0.29$)$ \\
MS symptoms, all follow up & $-7.82(-10.82$ to -4.82$)$ & $-4.52(-7.49$ to -1.56$)$ & $-3.30(-7.41$ to 0.81$)$ \\
MS symptoms, treatment compliant & $-9.64(-12.77$ to -6.51$)$ & $-5.19(-8.16$ to -2.21$)$ & $-4.45(-8.71 \text { to }-0.19)^{\text {b }}$ \\
MS ADL, all follow up & $1.59(-0.41$ to 3.59$)$ & $1.58(-0.43$ to 3.58$)$ & $0.01(-2.72$ to 2.75$)$ \\
MS ADL, treatment compliant & $1.86(-0.36$ to 4.09$)$ & $2.18(0.00$ to 4.35$)$ & $-0.31(-3.35$ to 2.73$)$ \\
Fatigue, all follow up & $-2.14(-2.81$ to -1.47$)$ & $-1.16(-1.83$ to -0.5$)$ & $-0.98(-1.89 \text { to }-0.06)^{\mathrm{c}}$ \\
Fatigue, treatment compliant & $-2.52(-3.21$ to -1.83$)$ & $-1.56(-2.22$ to -0.9$)$ & $-0.96(-1.89 \text { to }-0.03)^{d}$ \\
\hline
\end{tabular}

Values are adjusted means with $95 \%$ confidence intervals, and estimate of difference. ${ }^{a} \mathrm{p}=0.041 ;{ }^{b} \mathrm{p}=0.041 ;{ }^{\mathrm{c}} \mathrm{p}=0.037 ;{ }^{d} \mathrm{p}=0.044$.

ADL, activities of daily living; GNDS, Guy's neurological disability scale; MS, multiple sclerosis. 


\begin{tabular}{lll} 
Table 4 & Side effects observed & \\
\hline Group & Combination (n (\%)) & Control (n (\%)) \\
\hline Total & 69 & 69 \\
Constipation & $32(46)$ & $10(14)$ \\
Mouth dry & $22(32)$ & $7(10)$ \\
Nausea & $13(19)$ & $6(9)$ \\
Insomnia & $13(19)$ & $4(6)$ \\
Depression & $12(17)$ & $9(13)$ \\
Headache & $10(14)$ & $13(19)$ \\
Palpitations & $9(13)$ & $2(3)$ \\
Urinary tract infections & $9(13)$ & $14(20)$ \\
Fatigue & $8(12)$ & $12(17)$ \\
Hot flushes & $8(12)$ & $1(1.5)$ \\
Diarrhoea & $2(3)$ & $7(10)$ \\
Back pain & $2(3)$ & $6(9)$ \\
\hline
\end{tabular}

\section{Conclusions}

We conclude that there is insufficient evidence to support the use of this triple combination therapy routinely in patients with multiple sclerosis. There are trends suggesting some symptomatic improvement, and a small beneficial effect cannot be ruled out. Consequently further studies may be justified.

\section{ACKNOWLEDGEMENTS}

We gratefully acknowledge Scotia Pharmaceuticals who provided all the necessary funding, took responsibility for all aspects of data collection, and undertook preliminary analysis. We specifically thank Dr G Cooper, Dr R Bryce, Ms K McGregor, Ms B Attwood, and Dr B Patel. We thank all the patients for agreeing to participate, and Dr B Al-Saffar and other assessors who helped collect the data. We thank Professor M Barnes and Mrs Q Green for their help in the planning phases. We thank Professor Graham Dunn and Professor Martin Crowder for agreeing to help with the data analysis. We thank Dr Michael Johnson for helping to recruit patients and for running the project in Leeds and Dr R B Forbes and Mrs S McDonald for help given in Dundee.

\section{DISCUSSION}

This study in a group of patients with disability arising from multiple sclerosis showed a small reduction in cumulative disability over 24 weeks while they were taking combination triple therapy of L-phenylalanine, lofepramine, and vitamin B-12 (the Cari Loder regime). There was also a suggestion that patients who complied fully may have gained some increased relief from their symptoms. It was notable that both groups of patients experienced a reduction in disability over the first two weeks of the treatment. The further benefit was probably clinically insignificant and does not support the widespread use of this treatment, but it does suggest a need for further studies to investigate how the benefit arises.

This study had several strengths. It was large enough to detect a clinically relevant decrease in disability. In contrast to most other current studies of treatments for patients with multiple sclerosis, it included a wide spectrum of disability, with some very disabled patients, and covered a broad age range. Compliance was good. The range of measures used should have detected any major benefit in any one restricted domain.

There were some weaknesses. One potential criticism of this study is that it did not select the "correct" patients (that is, those who would benefit). In the absence of any scientific basis for the treatment and any published data to help identify such patients, this was not possible. An unvalidated checklist is available, but our study was not sufficiently large to allow this to be further assessed. The study might be criticised as using an inappropriate dose, but it explicitly tested the regime described in the lay press and used in Scandinavian practice. ${ }^{6}$ We did detect side effects which might be anticipated from the component drugs, suggesting that the combination therapy had some physiological effects. It is possible that the treatment helps certain specific symptoms or specific patients, but it was not powered to detect a selective or small benefit.

The results from the secondary analyses need to be interpreted very cautiously. The differences noted were small, the groups of treatment compliant patients are potentially biased, and when multiple comparisons are made some differences may arise through chance. At most one might conclude that combination therapy reduces symptoms, including fatigue, by a very small amount, but not sufficiently to reduce disability or influence psychological state (as measured by reported symptoms or depression).

This treatment study is one of the first to use the Guy's neurological disability scale ${ }^{7}$ as its primary outcome measure. The GNDS is a new scale specifically designed for use in trials, and we have found it to be easy to apply and well accepted by patients. It represents a great improvement over the Kurtzke EDSS, which has been criticised for insensitivity. ${ }^{16}{ }^{17} \mathrm{~A}$ postal version of the GNDS is now also available. ${ }^{9}$

(...................

Authors' affiliations

D T Wade, Oxford Centre for Enablement, Oxford, UK

C A Young, The Walton Centre for Neurology and Neurosurgery, Liverpool, UK

K R Chaudhuri, Department of Neurology, Lewisham and King's College Hospitals, London SE 13, UK

D L W Davidson, Department of Neurology, Ninewells Hospital, Dundee, UK

Contributors: CY and DW both took an active part in all aspects of the study including helping with the design and execution of the study, recommending the measures, and undertaking some of the data analysis. CY and DW are the guarantors. RC and DD took part in discussions about the design of the trial and in data interpretation, and checked the manuscript.

Competing interests: All investigating centres received some financial support to carry out the study, and investigators had expenses reimbursed.

\section{REFERENCES}

1 Clegg A, Bryant J, Milne R. Disease-modifying drugs for multiple sclerosis: a rapid and systematic review. Health Technol Assess 2000;4: i-iv, $1-10$.

2 Branas $\mathbf{P}$, Jordan $\mathrm{R}$, Fry-Smith $\mathrm{A}$, et al. Treatments for fatigue in multiple sclerosis. Health Technol Assess 2000;4:1-61

3 Freeman JA, Langdon DW, Hobart JC, et al. The impact of inpatient rehabilitation on progressive multiple sclerosis. Ann Neurol 1997:42:236-44.

4 Di Fabio RP, Soderberg J, Choi T, et al. Extended outpatient rehabilitation: its influence on symptom frequency, fatigue, and functional status for persons with progressive multiple sclerosis. Arch Phys Med Rehabil 1998:79:141-6.

5 Loder C. Standing in the sunshine: the story of the multiple sclerosis breakthrough. London: Century, 1996.

6 Berne-Fromell K, Fromell H, Lundkvist S, et al. Is multiple sclerosis the equivalent of Parkinson's disease for noradrenaline? Med Hypoth 1987:23:409-15.

7 Sharrack B, Hughes RAC. The Guy's Disability Scale: a new disability measure for multiple sclerosis. Multiple Sclerosis 1999;5:223-33.

8 Tubridy N, Behan PO, Capildeo R, and The UK Antegren Study Group. The effect of anti- $\alpha 4$ integrin antibody on brain lesion activity in MS. Neurology 1999;53:466-72.

9 Rossier P, Wade DT. The Guy's Neurological Disability Scale in patients with multiple sclerosis: a clinical evaluation of its reliability and validity. Clin Rehabil 2002:16:75-95.

10 Poser CM, Paty DW, Scheinberg L, et al. New diagnostic criteria for multiple sclerosis: guidelines for research protocols. Ann Neurol 1983;13:227-31.

11 Kurtzke JF. Rating neurological impairment in multiple sclerosis: an expanded disability status scale (EDSS). Neurology 1983;33:1444-52.

12 Beck AT, Ward CH, Mendelson $M$, et al. An inventory for measuring depression. Arch Gen Psychiatry 1961;31:122-6.

13 Chalder T, Berelowitz G, Pawlikowska T, et al. Development of a fatigue scale. J Psychosom Res 1993;37:147-53.

14 Gulick EE. Model confirmation of the MS-related symptom checklist. Nursing Res 1989;38:147-53.

15 Fuller KJ, Dawson K, Wiles CM. Physiotherapy in chronic multiple sclerosis: a controlled trial. Clin Rehabil 1996;10:195-204.

16 Sharrack B, Hughes RAC. Clinical scales for multiple sclerosis. I Neurol Sci 1996;135: 1-9.

17 Vaney C, Blaurock H, Gatter B, et al. Assessing mobility in multiple sclerosis using the Rivermead Mobility Index and gait speed. Clin Rehabil $1996 ; 10: 216-26$. 